\title{
Impact of Imperfect Beam Alignment on the Rate-Positioning Trade-Off
}

\author{
Giuseppe Destino*, Jani Saloranta*, Henk Wymeersch ${ }^{\dagger}$, and Gonzalo-Seco Granados ${ }^{\ddagger}$ \\ ${ }^{*}$ University of Oulu, Oulu, Finland \\ ${ }^{\dagger}$ Chalmers University of Technology, Gothenburg, Sweden \\ ${ }^{\ddagger}$ Universitat Autonoma de Barcelona, Barcelona, Spain \\ email: \{giuseppe.destino,jani.saloranta\}@oulu.fi, henkw@chalmers.se, gonzalo.seco@uab.cat
}

\begin{abstract}
We consider the beam-training procedure in the future 5G millimeter-wave systems and collect position information based on the received signals. We analyze the degradation due to beam misalignment on the achievable rate and on the amount of information available for positioning. We evaluate the performance of two beam-training strategies, namely, exhaustive and hierarchical. Our results reveal new insights on the trade-off between positioning and communication performance.
\end{abstract}

\section{INTRODUCTION}

Communication above $28 \mathrm{GHz}$, or so-called millimeter wave $(\mathrm{mmW})$ communication, is considered to be an enabler for high throughput in 5G [1] as well as for accurate positioning [2]. Due to the large available bandwidth, coupled with the possibility of packing massive antenna arrays in a small form-factor, mmW technology has the potential to make possible rates of tens of Gbps, by using massive multipleinput-multiple-output (MIMO) and transmit and receive beamforming [1], [3], [4]. Many research studies as well as 5G prototypes [5] are focusing on this new technology in order to understand and verify its real performance.

While large arrays have the potential to synthesize fine beams to increase the signal-to-noise ratio (SNR), even small pointing errors lead to severe SNR degradations [6]. This problem manifests itself most explicitly during beam alignment procedures, where on the one hand fast but simple procedures may lead to pointing errors, and on other hand slow procedures may lead to a reduction in spectral efficiency. To address this conflicting behavior, fast and accurate beam alignment procedures have to be devised. Several proposals have been put forth in recent years, [7]-[9]. For an overview, we refer the reader to [10], where several beamtraining strategies have been studied and compared based on the required overhead. Among the most widely used, it is worth mentioning the exhaustive and hierarchical techniques. The former is relevant for antennas capable of beamforming to fixed directions (e.g., lenses), whereas the latter is considered very efficient for reconfigurable antenna systems [5]. Beamtraining can be improved in terms of both speed and SNR when location information (e.g., from GPS) is available [11].

As $\mathrm{mmW}$ has the dual use of communication and positioning, it is then natural to analyze the trade-offs and synergies during initial access. Building on [12], [13], we quantify the trade-off between the data rate and positioning accuracy, as

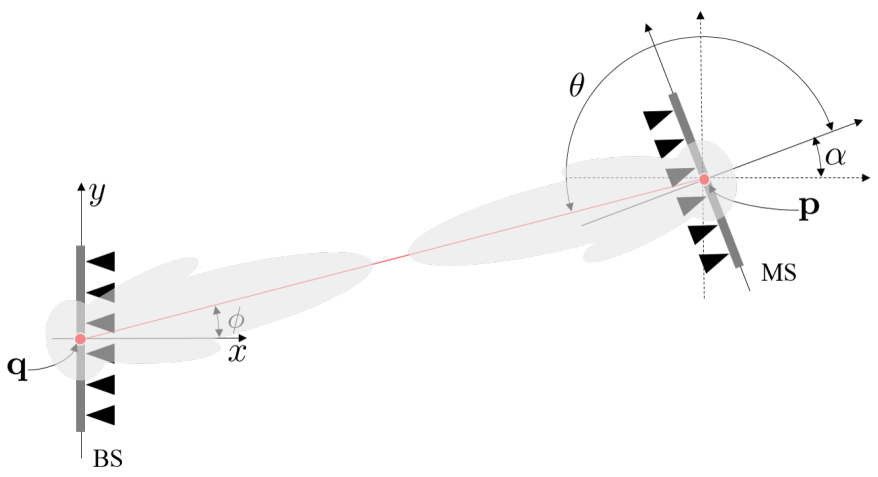

Figure 1. Geometry of the communication system including a receiver with fixed location and orientation, and a transmitter with unknown location $\mathbf{p}$ and orientation $\alpha$.

we vary the training overhead. In contrast to [12], [13], we account for the wrong beam decisions that will occur during the beam alignment process. We show that for the hierarchical search, when the initial SNR (i.e., in the first steps of alignment procedure) is low, both data rate and positioning accuracy are severely affected. In contrast, the exhaustive search is only affected in terms of the data rate, but not the positioning accuracy.

\section{MMW SINGLE-USER MIMO SYSTEM}

\section{A. Communication model}

We consider the up-link communication model for single mobile station (MS) in line-of-sight (LOS) channel condition with beamforming both at the transmitter and receiver sides, as in Figure 1. The received signal at the base station (BS) is given by

$$
y(t)=\sqrt{P_{\mathrm{tx}}} h \mathbf{w}^{\mathrm{H}} \mathbf{a}_{M}(\theta) \mathbf{a}_{N}^{\mathrm{H}}(\phi) \mathbf{f} x(t-\tau)+\mathbf{w}^{\mathrm{H}} \mathbf{n}(t),
$$

where $\mathbf{f} \in \mathbb{C}^{N}$ and $\mathbf{w} \in \mathbb{C}^{M}$ are the transmit and receive unit-norm beamforming vectors, $\mathbf{n}(t)$ is the additive white gaussian noise (AWGN) with power spectral density (PSD) $N_{0}, \tau$ is the time-delay of the LOS path, $x(t)$ represents one orthogonal frequency-division multiplexing (OFDM) symbol of the continuous time-domain transmitted signal of bandwidth $B$ and duration $T_{\text {sym }}$, with $1 / T_{\text {sym }} \int_{0}^{T_{\text {sym }}}|x(t)|^{2} \mathrm{~d} t=1, h \in \mathbb{C}$ is the LOS channel coefficient, $P_{\mathrm{tx}}$ is the transmission power, 
$\mathbf{a}_{N}(\phi) \in \mathbb{C}^{N}$ and $\mathbf{a}_{M}(\theta) \in \mathbb{C}^{M}$ are the receive and transmit array response vectors for the angle-of-departure (AoD) $\phi$ and angle-of-arrival (AoA) $\theta$ of the LOS path.

Using a geometry-based approach, the channel parameters (angles and path-delay) can be expressed as a function of the receiver's and transmitter's locations, i.e., $\tau=\|\mathbf{q}-\mathbf{p}\| / c$, $\theta=\operatorname{asin}\left(\left(q_{y}-p_{y}\right) /\|\mathbf{q}-\mathbf{p}\|\right), \theta=\pi-\alpha+\phi$, with $\alpha$ indicating the relative rotation between the transmit and receiving antenna arrays and $\mathbf{q}, \mathbf{p} \in \mathbb{R}^{2}$ denoting the receiver's (BS) and transmitter's (MS) location, respectively. For simplicity, but without any loss of generality, we consider a two-dimensional scenario. We assume that $\mathbf{q}$ is known, whereas $\mathbf{p}$ is not.

Both at the transmitter and receiver, we use uniform linear arrays (ULA's) with isotropic elements and $d=\lambda / 2$ element separation, where $\lambda$ is the carrier wavelength. Hence, the array response vector $\mathbf{a}_{M}(\theta)$, and similarly $\mathbf{a}_{N}(\phi)$, is given by

$$
\left[\mathbf{a}_{M}(\theta)\right]_{m}=e^{j \frac{2 \pi d}{\lambda}(m-1) \sin (\theta)}, m \in\{1, \ldots, M\} .
$$

Communication occurs over frames of duration $T_{r}=$ $N_{r} T_{\mathrm{sym}}$, a fraction $T_{t}=N_{t} T_{\mathrm{sym}}$ of which is devoted to beamtraining, where $N_{t} \leq N_{r}, N_{r}, N_{t} \in \mathbb{N}_{+}$are the number of OFDM symbols in a frame and number of symbols dedicated to training, respectively. The remainder $T_{d}=T_{r}-T_{t}$ is used for data transmission. Note that the best choice for w and $\mathbf{f}$ will depend of $N_{r}$ and $N_{t}$. As in [12], we assume that the training period is also used for positioning, i.e., for estimating the location $\mathbf{p}$ and orientation $\alpha$ of the transmitter. The objective of this work is to extend the results in [13] considering the effect of the noise on the search strategies. More specifically, we are interested in the characterization of the effective-rate as well as position error bound (PEB) when the noise causes errors in the beam alignment.

\section{B. Performance metrics}

The performance metrics used in this work are:

- effective data-rate: Let $\mathbf{w}$ and $\mathbf{f}$ be the beam pair selected after the beam-alignment. The effective data rate $R$ is

$$
R(\mathbf{w}, \mathbf{f})=B\left(1-\frac{T_{t}}{T_{r}}\right) \log _{2}\left(1+\frac{|h|^{2} P_{\mathrm{tx}} \mathrm{S}(\mathbf{w}, \mathbf{f}, \theta, \phi)}{\sigma^{2}}\right),
$$

in which the optimal beams ${ }^{1}$ are those that maximize the SNR:

$$
\left[\mathbf{w}^{*}, \mathbf{f}^{*}\right]=\arg \max _{\substack{\mathbf{w} \in \mathcal{W} \\ \mathbf{f} \in \mathcal{F}}} \mathrm{E}\left\{\frac{|h|^{2} P_{\mathrm{tx}} \mathrm{S}(\mathbf{w}, \mathbf{f}, \theta, \phi)}{\sigma^{2}}\right\} .
$$

where $\mathcal{W}$ and $\mathcal{F}$ are sets of all possible beamforming vectors for receiver and transmitter, respectively, $\mathrm{E}\{\cdot\}$ indicates the expected value, $S(\mathbf{w}, \mathbf{f}, \theta, \phi) \triangleq$ $\left|\mathbf{w}^{H} \mathbf{a}_{M}(\theta)\right|^{2}\left|\mathbf{f}^{H} \mathbf{a}_{N}(\phi)\right|^{2}, \sigma^{2}=N_{0} B$ is the noise power over the signal bandwidth.

- position-rotation error bound (PREB): Let $\boldsymbol{\xi} \triangleq\left[\mathbf{p}^{\mathrm{T}}, \alpha, h\right]^{\mathrm{T}}$ be the vector of unknown parameters to be estimated from the receiving signal $y(t)$. The PREB [12] is defined as

\footnotetext{
${ }^{1}$ Note that for a fixed $T_{t}$, the rate is maximized when $\mathbf{f}=1 / \sqrt{N} \mathbf{a}_{N}(\phi)$ and $\mathbf{w}=1 / \sqrt{M} \mathbf{a}_{M}(\theta)$.
}

the lower bound on the variance of the estimate $\hat{\mathbf{p}}^{\mathrm{T}}$ and $\hat{\alpha}$ and it comprises of two components: the position error bound

$$
\operatorname{PEB}=\sqrt{\operatorname{trace}\left(\left[\mathbf{J}_{\xi}^{-1}\right]_{1: 2,1: 2}\right)}
$$

expressed in meters, and the rotation error bound

$$
\mathrm{REB}=\sqrt{\left[\mathbf{J}_{\boldsymbol{\xi}}^{-1}\right]_{3,3}},
$$

expressed in radians (or degrees, after conversion), where

$$
\mathbf{J}_{\boldsymbol{\xi}}=\sum_{\ell=1}^{L} \mathbf{J}_{\boldsymbol{\xi}}^{\ell}\left(\mathbf{f}_{\ell}, \mathbf{w}_{\ell}\right)
$$

is the cumulative Fisher Information Matrix (FIM) associated to $\xi$ and obtained by aggregating $L$ FIM's, each one corresponding to the $\ell$-th beam-pair transmission $\left(\mathbf{f}_{\ell}, \mathbf{w}_{\ell}\right)$.

\section{NOISE IMPACT ON RATE AND PREB}

The expression in (3) shows that the effective data-rate is determined by the training overhead as well as by the choice of the beam-pair $(\mathbf{w}, \mathbf{f})$ for data transmission. If error occurs in the selection of $(\mathbf{w}, \mathbf{f})$, then it is expected that $R$ will decrease. Thus, the focus of this section is to model the average effective data-rate and average FIM, where the average is computed with respect to the decisions made during beamtraining, which depends on the specific beamtraining strategy.

\section{A. Average Effective Data-Rate}

Let $\pi_{q}$ denote the probability that a beam pair $\left(\mathbf{w}_{q}, \mathbf{f}_{q}\right)$ is selected for data transmission. The average effective data-rate is given by

$$
\bar{R}=B\left(1-\frac{T_{t}}{T_{r}}\right) \sum_{q=1}^{Q} \pi_{q} R\left(\mathbf{w}_{q}, \mathbf{f}_{q}\right),
$$

where $\mathbf{w} \in \mathcal{W}$ and $\mathbf{f} \in \mathcal{F}$ with the cardinality $|\mathcal{W}|$ and $|\mathcal{F}|$ being finite and $Q$ is the number of all beam-pair combinations.

Clearly, the probability $\pi_{q}$ is a function of multiple factors, such as beamforming codebook, beamtraining strategy, beamselection criteria as well as the channel model. Hereafter, we focus on the received signal strength beam selection method to derive the expression ${ }^{2}$ of $\pi_{q}$, under exhaustive and binary hierarchical beamtraining strategies.

Let $|Y|_{q}^{2}$ denote the received signal with the beam pair $\left(\mathbf{w}_{q}, \mathbf{f}_{q}\right)$, that is,

$$
|Y|_{q}^{2}=\frac{1}{T_{\mathrm{sym}}} \int_{0}^{T_{\mathrm{sym}}}\left|y_{q}(t)\right|^{2} \mathrm{~d} t
$$

where $y_{q}(t)=\sqrt{P_{\mathrm{tx}}} h \mathbf{w}_{q}^{\mathrm{H}} \mathbf{a}_{M}(\theta) \mathbf{a}_{N}^{\mathrm{H}}(\phi) \mathbf{f}_{q} x(t-\tau)+\mathbf{w}_{q}^{\mathrm{H}} \mathbf{n}(t)$.

\footnotetext{
${ }^{2}$ Note that we provide only the general formulation and simulation results.
} The derivation of the exact expressions is beyond the scope of the paper. 
We can express the probabilities for the exhaustive and hierarchical search strategies, respectively, as

$$
\begin{aligned}
& \pi_{q}=\prod_{p \neq q}^{P} \operatorname{Pr}\left(|Y|_{q}^{2}>|Y|_{p}^{2}\right), \\
& \pi_{q}=\prod_{k=1}^{K} \prod_{p \neq q}^{P} \operatorname{Pr}\left(|Y|_{k, q}^{2}>|Y|_{k, p}^{2}\right),
\end{aligned}
$$

where $K$ is the total number of stages in the hierarchical search. The pairs of indexes $k, q$ and $k, p$ refer to the pair of transmit-receive beams used at the $k$-th stage in the $q$-th and $p$-th leaves [11], respectively. The value of $P$ is $P=Q-1$ in (10), while it is equal to the number of sector partitions in (11).

\section{B. Average FIM}

Unlike the rate, the positioning error is determined by the training symbols and not by data. Therefore, our objective is to characterize the errors that noise yields during this period and their effect in the collection of positioning information.

As explained in [12], [13], using the exhaustive search, positioning information is collected for all possible beampairs. Thus, the noise has simply an impact on the SNR level.

On the other hand, with the hierarchical search, the presence of noise may cause wrong decisions already at early stages. Therefore, the cumulative FIM may be, in some cases, totally uninformative.

Subsequently, this leads to the definition of the average FIM for the exhaustive and hierarchical search strategies, respectively, as

$$
\begin{aligned}
& \overline{\mathbf{J}}_{\boldsymbol{\xi}}=\sum_{q=1}^{Q} \pi_{q} \mathbf{J}_{\boldsymbol{\xi}}^{q}\left(\mathbf{w}_{q}, \mathbf{f}_{q}\right), \\
& \overline{\mathbf{J}}_{\boldsymbol{\xi}}=\sum_{k=1}^{K} \sum_{q=1}^{Q} \pi_{q} \mathbf{J}_{\boldsymbol{\xi}}^{q}\left(\mathbf{w}_{k, q}, \mathbf{f}_{k, q}\right),
\end{aligned}
$$

where $\pi_{q}$ in (12) is defined in (10) and in (13) is defined in (11), $\mathbf{w}_{k, q}$ and $\mathbf{f}_{k, q}$ are the beam pair at level $k$ for leaf $q$, and $Q$ is the total number of beam-pairs in (12), while in (13) $Q$ is equals to the number of sector partitions.

\section{Simulation Results}

We consider a MIMO-OFDM communication system, similar to scenarios investigated in [13], [14]. We assume 1200 subcarriers in use and $\Delta_{f}=75 \mathrm{kHz}$ for pilot and data transmission. The transmission power is fixed to $P_{\mathrm{tx}}=30 \mathrm{dBm}^{3}$; the BS and the MS have the same number of antennas, i.e., $N=M$; the carrier frequency is $28 \mathrm{GHz}$, and frame duration is fixed to $T_{r}=20 \mathrm{~ms}$, i.e., 1500 symbols per frame, then $T_{\text {sym }}=13.3 \mathrm{~ns}$. During the training, each OFDM symbol is associated to one beam direction (time-multiplexing). Therefore, using an exhaustive search, the maximum size of

\footnotetext{
${ }^{3}$ Assuming $30 \mathrm{dBm}$ transmission power, the EIRP for $N=16$ is $42 \mathrm{dBm}$ below $70 \mathrm{dBm}$ proposed for $5 \mathrm{G}$-base station and $43 \mathrm{dBm}$ for mobile [15]
}

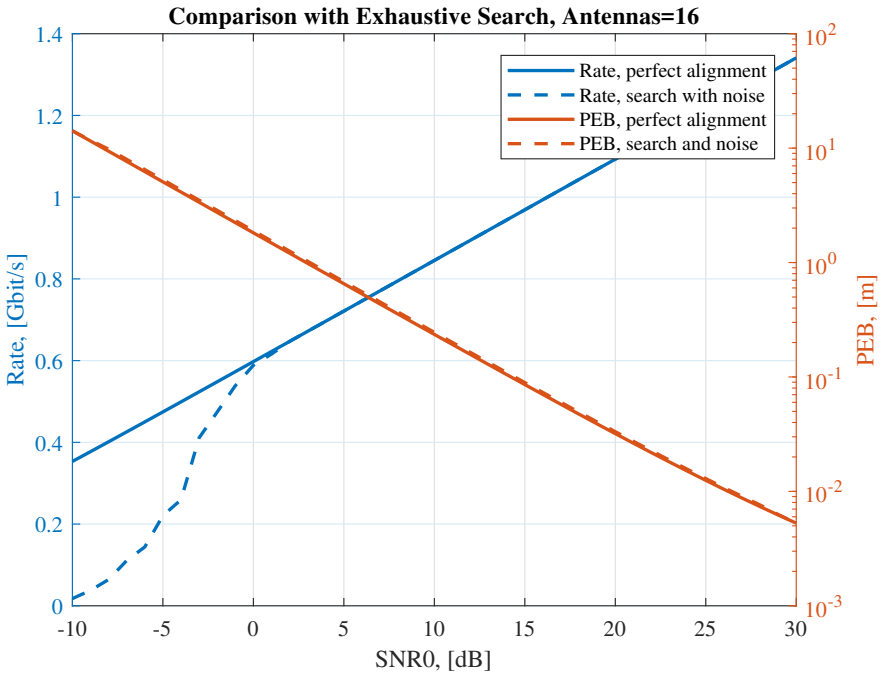

Figure 2. Comparison on the achievable rate (left axis) and position error bound (right axis) of exhaustive search strategy with perfect and imperfect beam-channel alignment. Number of antenna elements on BS and MS is equal $N=M=16$.

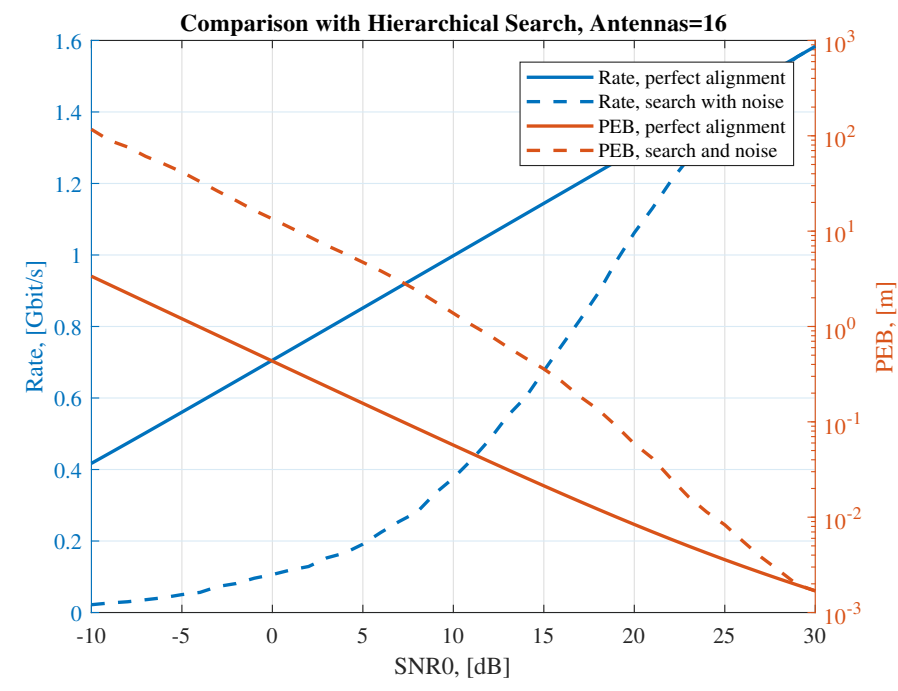

Figure 3. Comparison on the achievable rate (left axis) and position error bound (right axis) of hierarchical search strategy with perfect and imperfect beam-channel alignment. Number of antenna elements on BS and MS is equal $N=M=16$.

the transmitter and receiver codebook ${ }^{4}$ is $\lfloor\sqrt{1500}\rfloor=38$, i.e., $T_{t} / T_{r} \approx 1$.

For simplicity, the MS is in the boresight direction of the BS $\left(\phi=0^{\circ}\right)$, and the MS is aligned without rotation $\left(\alpha=0^{\circ}\right)$. The distance between BS and MS is adjusted to give a target $\mathrm{SNR}_{0}$, where $\mathrm{SNR}_{0}$ does not include the array gain factor. We assume a outdoor path-loss model with the power-decay coefficient 2.55. This yields an $\mathrm{SNR}_{0}=40 \mathrm{~dB}$ for $11 \mathrm{~m}$ and $\mathrm{SNR}_{0}=0 \mathrm{~dB}$ for $412 \mathrm{~m}$.

\footnotetext{
${ }^{4}$ Note that for hierarchical search based on ternary tree, the overhead for a
} similar type of beams is approximately $2.5 \%$. 


\section{A. Noise impact on effective data rate and PEB}

The results illustrated in Figures 2 and 3 show the achievable rate and PEB as a function of the $\mathrm{SNR}_{0}$ with the exhaustive and hierarchical training strategies, respectively. Both search strategies are investigated in a scenario where number of antenna elements are fixed to $N=M=16$ for both, BS and MS. The results are compared between two cases, perfect and imperfect beam alignment, corresponding to absence or presence, respectively, of decisions errors caused by the AWGN noise.

Let us focus first on the exhaustive search (Figure 2) and compare the case between the perfect alignment (solid line) and a search procedure affected by the AWGN noise (dashed line). It can be noticed that the achievable rate is only affected by the noise when $\mathrm{SNR}_{0}<0 \mathrm{~dB}$. On the other hand, the PEB does not show any deviation from the perfect alignment case. As indicated in Section III-B, this is due to the fact that the FIM if obtained with all beam-pairs and no decision on the beam selection is made during the training.

Next, we focus on the hierarchical search strategy in case of the perfect alignment and compare the rate in Figure 2 with the rate in Figure 3. Clearly, with no beam-alignment errors the effective rate is higher with hierarchical strategy than with exhaustive strategy thanks to lower training overhead.

On the other hand, when error occurs during the beam search in the hierarchical strategy, both rate and PEB degrade significantly. Also, the losses start when $\mathrm{SNR}_{0}<28 \mathrm{~dB}$, which is a much higher value than that in the exhaustive strategy. The reason is inherent to the search mechanism, since the beams used in step depend on the beam-selection decision taken in the previous step. Thus, if errors occurs at the beginning, final beams will be very far away from the optimal ones. Next we look into the aforementioned phenomena that is captured in Figure 4, which shows the probability selecting the optimal beam for both search strategies. The results are provided as a function of the SNR for two different cases of number of antennas, $N=M=8$ and $N=M=16$.

An interesting observation is that the hierarchical search yields lower probability when the number of antenna increases (i.e., when final beamwidth becomes increasingly narrow). This is, in fact, the consequence of having a larger number for stages, thus larger probability of making mistakes.

\section{B. Trade-off on data rate and positioning error}

In this Section we study the trade-off between positioning performance and effective data rate and the results are shown Figure 5.

As shown in [12], the trade-off depends on the training period duration, which is directly related to the codebook size, and thus to the number of antennas. The results are drawn for two SNR levels, namely $\mathrm{SNR}_{0}=20 \mathrm{~dB}$ and $\mathrm{SNR}_{0}=40 \mathrm{~dB}$.

In [13], we studied in detail the trade-off without beamalignment errors. In this case, for the hierarchical search strategy, there is not a trade-off as both metrics, data rate and $\mathrm{PEB}$, improves with the number of antennas.

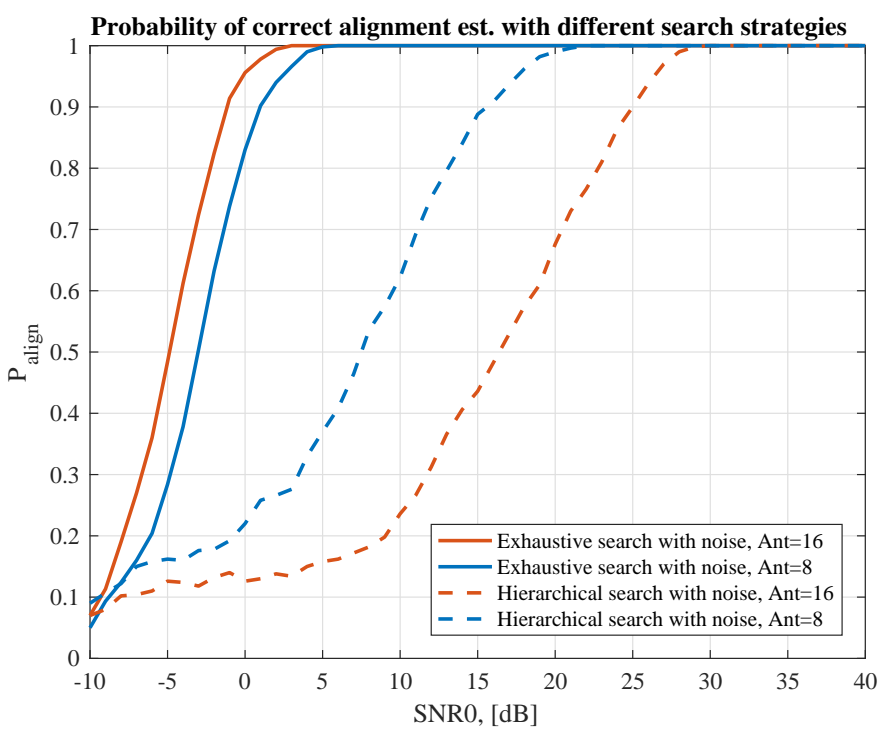

Figure 4. Probability of correct beam alignment in estimation as function of SNR for two different cases of number of antenna elements, 8,16 . Number of antenna elements on BS and MS is equal $N=M$.

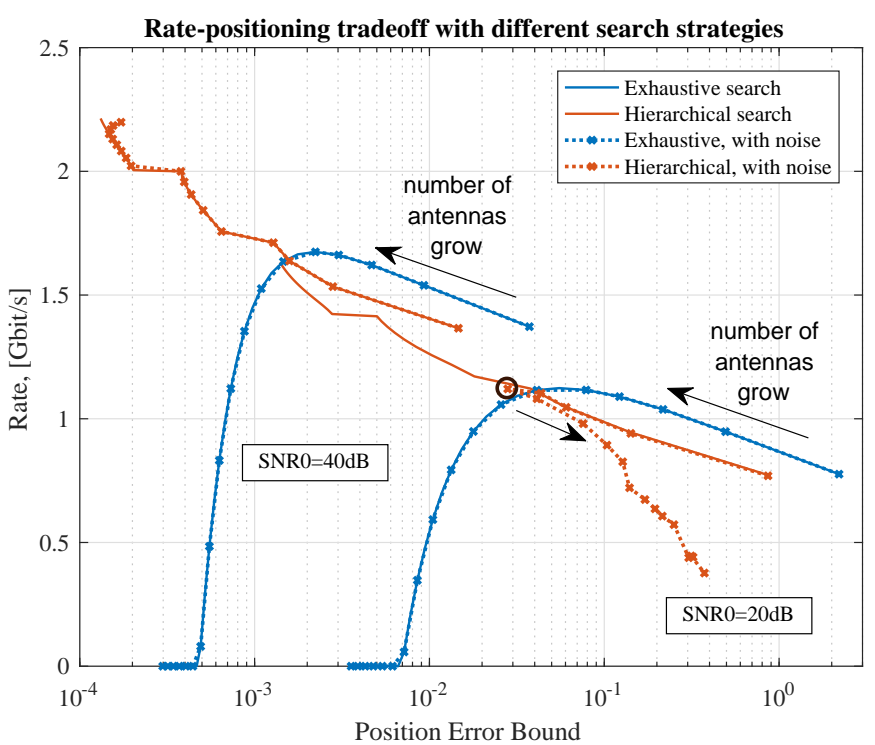

Figure 5. Rate as function of position error bound and number of antenna elements on BS and MS $N=M$. The black arrow indicating the direction in which the number of antennas is growing.

But, looking into hierarchical search strategy under the effect of AWGN noise, we can notice that having high SNR regime $\left(\mathrm{SRN}_{0}=40 \mathrm{~dB}\right)$ becomes a requirement to maintain the advantage on the exhaustive search. In fact, at $\mathrm{SRN}_{0}=20 \mathrm{~dB}$, we notice a turn-around point, where the performance begins to decrease in both, rate and PEB. The point is illustrated with black circle in Figure 5. This point reflects the presence of beam-alignment errors during the training phase, which implies rate decrease and PEB increase.

In contrast, with the exhaustive search strategy, we notice that the behavior in performance is similar in both $\mathrm{SRN}_{0}$ regimes, as well as in both scenarios with and without AWGN 
noise. This highlights the fact that there is no impact on the PEB and that increasing the number of antennas can improve both rate and PEB provided that the overhead is low.

\section{CONClusion}

We investigated the impact of noise-induced beam selection errors on the data rate and the positioning accuracy of two beamtraining strategies, exhaustive and hierarchical search strategy. One of the main outcomes is the assessment on the high sensitivity to noise of hierarchical search strategy. In fact, results shows that higher is the number of stages to go though, higher is a high probability of making mistakes. Therefore, all the advantages gained with the reduced training time are lost if no power-boosting mechanisms are utilized during the training. On the other hand, the exhaustive search showed more robustness to noise, but it can be prohibitive for the high overhead due to large number of beam-pairs to be sounded.

Future work will focus on a more realistic radio propagation model which includes multipaths. In that case, scatter/reflector locations might help the positioning performance, but yields a non-trivial trade-off with the rate.

\section{ACKNOWLEDGMENTS}

The research leading to these results has received funding from the Academy of Finland, Fundamental of Simultaneous Localization and Communications (FALCON), Academy of Finland, Positioning-aided Reliably-connected Industrial Systems with Mobile mmWave Access (PRISMA), as well as the European Union H2020 5GPPP under Grant No. 723247 and supported by the Institute for Information \& communications Technology Promotion (IITP) grant funded by the Korea government (MSIP) No. B0115-16-0001 (5GCHAMPION). This work is partially funded by the Spanish Ministry of Science and Innovation project TEC2014-53656-R. Henk Wymeersch was supported by the EU H2020 projects HIGHTS (High Precision Positioning for Cooperative ITS Applications, Grant No. MG-3.5a-2014-636537) and 5GCAR (Fifth Generation Communication Automotive Research and innovation, Grant No. 761510).

\section{REFERENCES}

[1] Z. Pi and F. Khan, "An introduction to millimeter-wave mobile broadband systems," IEEE Commun. Mag., vol. 49, no. 6, pp. 101-107, Jun. 2011.

[2] A. Shahmansoori, G. E. Garcia, G. Destino, G. Seco-Granados, and H. Wymeersch, "Position and Orientation Estimation through Millimeter Wave MIMO in 5G Systems," ArXiv e-prints, Feb. 2017.

[3] P. Wang, Y. Li, L. Song, and B. Vucetic, "Multi-gigabit Millimeter Wave Wireless Communications for 5G: From Fixed Access to Cellular Networks," IEEE Commun. Mag., vol. 53, no. 1, pp. 168-178, Jan. 2015.

[4] R. W. Heath, N. González-Prelcic, S. Rangan, W. Roh, and A. M. Sayeed, "An Overview of Signal Processing Techniques for Millimeter Wave MIMO Systems," IEEE J. Sel. Topics Signal Process., vol. 10, no. 3, pp. 436-453, Apr. 2016.

[5] M. Mueck et al., "5G CHAMPION - Rolling out 5G in 2018," in Proc. IEEE Global Commun. Conf. Workshops, Dec. 2016, pp. 1-6.

[6] S. Hur, T. Kim, D. J. Love, J. V. Krogmeier, T. A. Thomas, A. Ghosh et al., "Millimeter wave beamforming for wireless backhaul and access in small cell networks." IEEE Trans. Communications, vol. 61, no. 10, pp. 4391-4403, 2013
[7] Z. Xiao, T. He, P. Xia, and X. G. Xia, "Hierarchical Codebook Design for Beamforming Training in Millimeter-Wave Communication," IEEE Trans. Wireless Commun., vol. 15, no. 5, pp. 3380-3392, May 2016.

[8] C. Liu, M. Li, I. B. Collings, S. V. Hanly, and P. Whiting, "Design and Analysis of Transmit Beamforming for Millimeter Wave Base Station Discovery," IEEE Trans. Wireless Commun., vol. 16, no. 2, pp. 797-811, Feb. 2017.

[9] B. W. Ku, D. G. Han, and Y. S. Cho, "Efficient Beam-Training Technique for Millimeter-Wave Cellular Communications," ETRI Journal, vol. 38, no. 1, pp. 81-89, 2016.

[10] S. Kutty and D. Sen, "Beamforming for Millimeter Wave Communications: An Inclusive Survey," IEEE Commun. Surveys Tuts., vol. 18, no. 2, pp. 949-973, 2016

[11] N. Garcia, H. Wymeersch, E. G. Ström, and D. Slock, "Locationaided mm-wave channel estimation for vehicular communication," in IEEE International Workshop on Signal Processing Advances in Wireless Communications (SPAWC), Jul. 2016, pp. 1-5.

[12] G. Destino and H. Wymeersch, "On the Trade-off Between Positioning and Data-Rate for mm-Wave Communications," in Proc. IEEE Int. Conf. Commun. Workshop, May 2017.

[13] J. Saloranta, G. Destino, and H. Wymeersch, "Comparison of Different Beamtraining Strategies from A Rate-positioning Trade-off Perspective," in Proc. Eur. Conf. Networks and Commin., Jun. 2017, pp. 1-5.

[14] J. Saloranta and G. Destino, "Reconfiguration of $5 \mathrm{~g}$ radio interface for positioning and communication," in Proc. European Sign. Process. Conf., Aug. 2017, pp. 898-902.

[15] FCC, "Fact sheet: Spectrum frontiers rules identify, open up vast amounts of new high-band spectrum for next generation $(5 \mathrm{~g})$ wireless broadband," Tech. Rep., Jul. 2016. 